\title{
Kräutermixtur gegen Nahrungsmittelallergien?
}

Ein Kräuterextrakt aus der traditionellen chinesischen Medizin schützt Mäuse mit Erdnussallergie vor erdnussinduzierter Anaphylaxie. In einer kurzen Phase-I-Studie hatte sich die Mixtur auch beim Menschen als sicher und verträglich erwiesen. Jetzt liegen Daten aus der Verlängerungsphase vor.

ei dem untersuchten Präparat FAHF-

2 (für Food Allergy Herbal Formu-

la-2) handelt es sich um einen Extrakt aus neun verschiedenen Kräutern. Die PhaseI-Studie mit FAHF-2 dauerte nur eine Woche, anschließend wurde sechs Monate lang mit dreimal täglich sechs Tabletten $(3,3 \mathrm{~g})$ offen weiterbehandelt. Alle 18 Studienteilnehmer hatten eine nachgewiesene Allergie gegen Erdnüsse und/oder Nüsse.

Vier Patienten beendeten die Studie vorzeitig. Bei den 14 Patienten (zwölf bis 27 Jahre), die bis zum Ende dabei blieben, war der Kräuterextrakt auch langfristig sicher und gut verträglich. Während der sechsmonatigen Behandlung blieben Laborparameter, Lungenfunktion und EKG bei allen Probanden unverändert. Keiner der Studienteilnehmer erlebte - unter seiner üblichen Eliminationsdiät - eine lebensmittelinduzierte Allergie.
Weitere Untersuchungen lieferten Hinweise, dass FAHF-2 auch beim Menschen die Immunantwort auf (Erd-)Nussallergene dämpfen könnte. Ex-vivo-Provoka-

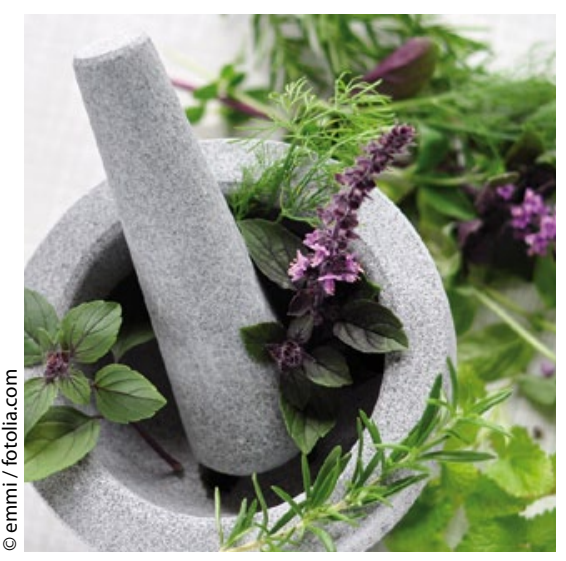

Ein Extrakt aus neun verschiedenen Kräutern wurde getestet. tionen mit $200 \mathrm{pg} / \mathrm{ml}$ eines spezifischen Allergenextrakts induzierten bei Studienende eine schwächere Aktivierung der basophilen Granulozyten - gemessen über die CD63-Expression von CCR3positiven Zellen - als bei Studienbeginn. Darüber hinaus verminderte sich unter der Therapie auch die Zahl der Basophilen (von 0,68 auf 0,39\%) und der Eosinophilen im peripheren Blut (von 5,3 auf $3 \%)$. Die Unterschiede waren allerdings nur grenzwertig signifikant.

Fazit: Der Kräuterextrakt FAHF-2 zeigte sich auch in der Verlängerung einer Phase-I-Studie als sicher und gut verträglich für Patienten mit Nahrungsmittelallergie. Außerdem gingen unter der Behandlung die Aktivität und der Anteil der Basophilen zurück. Die Ergebnisse sollen jetzt in einer Phase-II-Studie überprüft werden.

Dr. Beate Schumacher

Patil SP et al. Clinical safety of Food Allergy Herbal Formula-2 (FAHF-2) and inhibitory effect on basophils form patients with food allergy: extended phase I study. J Allergy Clin Immunol 2011; 128: 1259-65

\section{Was bringt die Interleukin-5-Hemmung bei eosinophiler Ösophagitis?}

Die Therapie der eosinophilen Ösophagitis ist vor allem bei Kindern und Jugendlichen problematisch. Denn die üblichen Glukokortikoide können unter anderem das Wachstum verzögern. US-Forscher haben nun versucht herauszufinden, ob den Betroffenen mit dem Interleukin-5-Hemmer Reslizumab zu helfen ist. Ergebnis: teils, teils.

E rkenntnisse aus Tierversuchen legen nahe, dass Interleukin 5 (IL-5) die eosinophile Entzündung im Ösophagus befeuert. Reslizumab ist ein monoklonaler Antikörper, der in der Lage ist, IL-5 zu neutralisieren. Daher könnte sich mit der Substanz auch der Verlauf der eosinophilen Ösophagitis beeinflussen lassen.

An einer entsprechenden randomisierten, doppelt verblindeten und placebokontrollierten Studie beteiligten sich 226 Patienten im Alter von fünf bis 18 Jahren. Rund $90 \%$ von ihnen wiesen klinisch mäBige bis sehr schwere Symptome auf, wie etwa Schmerzen, Erbrechen oder Schluckbeschwerden. Im Median waren in den
Gewebeproben der Speiseröhre 80 intraepitheliale Eosinophile pro Hauptgesichtsfeld (400-fache Vergrößerung, 0,3 mm²) $\mathrm{zu}$ finden. $\mathrm{Zu}$ Beginn der Studie sowie nach vier, acht und zwölf Wochen erhielten die Probanden 1, 2 oder 3 mg/kg Reslizumab oder eine Placebolösung als Infusion. Nach 15 Wochen wurden die Gewebseosinophilen erneut gezählt. Außerdem wurde der klinische Befund erhoben und anhand des „Physician's Eosinophilic Esophagitis Global Assessment“ einem von fünf Graden zugeordnet.

Tatsächlich hatte die Zahl der Eosinophilen bei jenen Probanden, die den IL5-Hemmer infundiert bekommen hatten, im Vergleich zu Placebo signifikant abgenommen. Die Reduktion betrug 59\% in der 1-mg-, $67 \%$ in der 2-mg- und $64 \%$ in der 3-mg-Gruppe. Auch klinisch ergaben sich Verbesserungen: Hatten zu Studienbeginn nur knapp $10 \%$ der Teilnehmer keine oder leichte Beschwerden, waren es nun 56,4\% (1 mg), 63,2\% (2 mg), 64,9\% (3 mg) bzw. 71,9\% (Placebo). Die Verumsubstanz schnitt hier aber nicht signifikant besser ab als Placebo.

Fazit: Reslizumab wirkt gegen eosinophile Ösophagitis von Kindern und Jugendlichen - zumindest unter dem Mikroskop. Klinisch bringt eine zwölfwöchige Intervalltherapie zunächst keine Vorteile gegenüber Placebo. $\mathrm{Ob}$ eine längerfristige Therapie besser abschneidet, wird zurzeit untersucht.

Dr. Robert Bublak

Spergel JM et al. Reslizumab in children and adolescents with eosinophilic esophagitis: Results of a double-blind, randomized, placebo-controlled trial. J Allergy Clin Immunol 2012; 129: 456-63 